\title{
Écrit de soi et combat politique. Les Mémoires de Jeanne d'Albret, reine de Navarre
}

\section{Bruno Tolaïni}

\section{(2) OpenEdition}

Journals

\section{Édition électronique}

URL : http://journals.openedition.org/rief/1023

DOI : 10.4000/rief.1023

ISSN : 2240-7456

\section{Éditeur}

Seminario di filologia francese

\section{Référence électronique}

Bruno Tolaïni, «Écrit de soi et combat politique. Les Mémoires de Jeanne d'Albret, reine de Navarre », Revue italienne d'études françaises [En ligne], 5 | 2015, mis en ligne le 15 décembre 2015, consulté le 04 mai 2019. URL : http://journals.openedition.org/rief/1023 ; DOI : 10.4000/rief.1023

Ce document a été généré automatiquement le 4 mai 2019.

\section{(c) (i) (9)}

Les contenus de la RIEF sont mis à disposition selon les termes de la Licence Creative Commons Attribution - Pas d'Utilisation Commerciale - Pas de Modification 4.0 International. 


\title{
Écrit de soi et combat politique. Les Mémoires de Jeanne d'Albret, reine de Navarre
}

\author{
Bruno Tolaïni
}

1 Reine de Navarre à la mort de son père en mai 1555, Jeanne d'Albret fut une figure emblématique de la vie politique et religieuse de l'Europe du XVI siècle. Elle fit de ses terres un bastion de la religion réformée grâce à une série de mesures dont la plus emblématique demeure la rédaction des Ordonnances ecclésiastiques en 1566 et 1571, imposant de fait le principe de Cujus regio, ejus religio ${ }^{1}$. Si l'historiographie traditionnelle a surtout retenu son austérité, sa ferveur religieuse et son opposition à la politique de Catherine de Médicis, il convient de rappeler que Jeanne était également une lettrée accomplie et qu'elle fut l'une des trois seules femmes mémorialistes du XVI ${ }^{\mathrm{e}}$ siècle $^{2}$. La souveraine avait en effet reçu une éducation humaniste approfondie sous le regard de Nicolas Bourbon et, à l'instar de sa mère Marguerite d'Angoulême, s'était plu à écrire. Outre une abondante correspondance, Jeanne est à l'origine de sonnets, rédigés pour la plupart avant le déclenchement des Guerres de religion, et de Mémoires ${ }^{3}$. Ce dernier texte n'a pas été rédigé sans raison; il devait empêcher la reprise des hostilités entre les catholiques et les protestants du royaume et ce qui fut finalement la troisième guerre de religion. Il s'agissait pour la reine de Navarre de dresser un violent réquisitoire contre ses ennemis à l'attention de Catherine de Médicis et de Charles IX et de justifier son implication nouvelle dans les conflits. Parce qu'il fut un moyen d'action, ce texte avait assurément une portée politique, il fallait déconsidérer l'adversaire. C'est précisément cet aspect des Mémoires de Jeanne d'Albret que nous nous proposons d'étudier aujourd'hui. À partir des choix rédactionnels faits par la mémorialiste pour se justifier et tenter de convaincre, nous tâcherons de comprendre de quelles manières le récit de soi peut intervenir sur la scène politique. 


\section{Morphologie d'un récit revendicatif}

2 Les Mémoires de Jeanne d'Albret ne ressemblent pas aux autres textes de la période et leur appartenance à un corpus de Mémoires du XVIe siècle n'est pas forcément évidente ${ }^{4}$. Rédigés dans l'urgence, ils ne contiennent pas les éléments essentiels de ces textes qui fondent la poétique du genre : les récits d'enfance, les triomphes, la disgrâce ou la clôture 5. Pour autant, le récit rétrospectif d'événements vus ou vécus que constitue le texte de la reine de Navarre nous semble pouvoir être qualifié de Mémoires. L'œuvre a été écrite à La Rochelle à la fin de l'année 1568 , après novembre puisque Jeanne parle des arrêts des parlements de Toulouse et de Bordeaux qui confisquaient ses biens, mais avant la fin décembre, date de l'ouverture des opérations militaires. La paix de Longjumeau qui mettait fin à la seconde guerre civile était alors mal appliquée et les combats menaçaient de reprendre à chaque instant. Les partis attendaient, l'arme au poing, l'occasion d'écraser le camp adverse. Dans l'espoir de faire changer le cours des événements qui se profilaient, Jeanne d'Albret composa, à l'attention de Catherine de Médicis et de Charles IX, un texte qui se voulait une amplification de tout ce qu'elle avait déjà exposé dans sa correspondance ${ }^{6}$. Le titre choisi, Ample déclaration, participe de la spécificité de ces Mémoires que la reine de Navarre n'envisageait probablement pas comme tels ${ }^{7}$. Dans les premières lignes du récit de sa vie, nous retrouvons les mêmes termes qui insistent encore sur les motivations de la mémorialiste: elle souhaitait "plus amplement faire entendre $»^{8}$, elle écrivait " pour amplifier $»^{9}$, elle voulait que « chacun entende $»^{10}$. Le tout est ostensiblement précédé du pronom Je, premier mot de l'œuvre, qui permit à Jeanne de revendiquer immédiatement la responsabilité de son écriture. Cette volonté d'être lue du plus grand nombre a de quoi surprendre : si l'autodestination radicale des Mémoires est à exclure, elle est souvent feinte et peu nombreux furent les mémorialistes de l'Ancien Régime qui avaient spécifiquement notifié leur souhait de diffuser leurs écrits ${ }^{11}$. Agir de cette manière permettait de gagner un temps précieux, dans le contexte de la reprise imminente des hostilités: Jeanne ne pouvait pas se permettre d'attendre que par indiscrétions le contenu de son récit fût connu de Catherine de Médicis et Charles IX. En outre, il s'agissait de prendre pour témoins tous ceux qui auraient ces feuilles entre les mains et qui devaient dès-lors pouvoir attester que la reine de Navarre avait exhorté la reine mère à maintenir la paix dans le royaume.

3 Le développement proposé par la reine de Navarre est composé de quatre parties ${ }^{12}$. Dans un premier temps, et dans un ordre propre à la rhétorique réformée qui privilégie d'abord le rapport entre le croyant et Dieu, Jeanne d'Albret évoqua sa foi. Sa conversion au protestantisme constitua le point de départ de son récit ${ }^{13}$. Ceci suggère qu'il s'agissait de l'élément déterminant de son opposition à la politique du royaume de France :

Je diray, commençant par la Religion, que, depuis l'an mil cinq cents soixante, il n'y a personne qui ne sçache bien qu'il pleut à Dieu par sa grâce me retirer de l'idolatrie, ou j'estoy trop plongée, et me recevoir en son Eglise. Depuis ce temps-là, par sa mesme grâce, il m'y a faict persévérer, de sorte que je me suis toujours employée à l'avancement d'icelle. ${ }^{14}$

Réformée depuis son abjuration au catholicisme en 1560, Jeanne se présenta ainsi avant tout comme une femme de constance qui était restée ancrée dans ses convictions malgré les troubles, et en dépit de l'apostasie de son époux ${ }^{15}$. Dans une seconde partie, la reine de Navarre chercha à décrédibiliser la maison de Guise et notamment le cardinal de Lorraine dans un développement bien plus étoffé que le premier. Il fallait également avancer les 
raisons qui avaient poussé les protestants dans la seconde guerre, de septembre 1567 à mars 1568, et dans la préparation du troisième conflit. Dans la troisième partie, Jeanne fit état de ses ultimes tentatives pour parvenir à une paix durant son voyage pour La Rochelle où elle rejoignit le prince de Condé, ainsi que le récit de ce périple. La quatrième et dernière partie, très brève, constituait un retour dans le présent de l'écriture et permit à la mémorialiste de théoriser et conclure son récit ${ }^{16}$. Elle expliqua entre autres choses que son écriture n'était en rien fardée et surtout elle mit en avant la vérité de son discours :

Seulement de la vérité, laquelle elle y a si fidèlement observée, qu'au moins, si elle est dicte ignorante et imbécille, elle sera dicte véritable. ${ }^{17}$

5 Cela permit également à Jeanne de nouer un pacte avec ses lecteurs, comme le firent la grande majorité des mémorialistes du temps, à la différence qu'ici il ne se trouve pas dans les premières pages de l'œuvre mais bien à la fin, proposant un schéma argumentatif atypique pour le genre ${ }^{18}$. En effet, alors que cet engagement de vérité se trouve presque toujours, comme le précise Nadine Kuperty-Tsur, dans la préface de ces œuvres, il fut ici proposé en postface ${ }^{19}$. Ces mots ont d'autant plus d'importance qu'ils clôturent le texte : tous les arguments vraisemblables précédemment développés par la mémorialiste pour justifier la prise d'armes des protestants et espérer une sincère inversion de la politique royale sont alors cachetés par le sceau de la vérité de la reine de Navarre.

\section{Un texte justificatif}

6 La justification que portent les Mémoires de Jeanne d'Albret est essentiellement contenue dans la seconde partie du découpage que nous avons proposé. Il fallait exposer synthétiquement toutes les accusations dont elle et l'ensemble des chefs protestants avaient pu être l'objet et s'en défendre, comme nous l'avons souligné, de manière vraisemblable, afin d'entraîner l'adhésion du lecteur. L'entreprise n'était pas simple: comment donner l'impression que l'on est favorable à la paix lorsque l'on prépare la guerre $^{20}$ ? Jeanne choisit de répondre une à une aux accusations dont elle fut la victime.

7 En tant qu'autorité temporelle des réformés et belle-sœur de Condé, Jeanne dut principalement se défendre de la charge de rébellion. La stratégie argumentative mise en place dans les Mémoires que nous étudions consistait à attaquer les principaux personnages de la maison de Guise, qui dirigeaient le clan adverse, pour se grandir et légitimer la prise d'armes des protestants. Le cardinal de Lorraine fut alors particulièrement malmené dans les propos de notre mémorialiste : barbare, malicieux, irrespectueux, traitre et manipulateur constituent autant de qualificatifs qui déprécient le prince de l'Eglise. La première des attaques lancées revenait sur l'élément déclencheur de la seconde guerre civile, la surprise de Meaux, que Jeanne justifia par la perfidie de ses opposants :

Je suis contrainct de redire encore une fois que c'est une chose trop incroyable que ce prince se soit laissé enchanter à eux, qui ont faict comme le peintre; lequel reblanchissant le tableau peint, efface ce qui y estoit, pour y mettre de nouveau ce qu'il a en affection. Car, par ces ruses susdictes, ils lui effacèrent la mémoire des lasches et méchants tous qu'ils luy avoyent faict, pour sur cet oubli repeindre leurs stratagèmes. ${ }^{21}$

8 L'influence des Guise à la cour serait à ce point dommageable au royaume de France qu'elle justifiait la traîtrise des réformés. Le cardinal de Lorraine était alors accusé de 
jouer «à la pelote de sa foy et son honneur $»^{22}$. De la même manière, lorsqu'il s'était agi d'évoquer l'armement des protestants dans le courant du mois d'août 1568, la reine de Navarre rendit responsable la maison de Guise :

Il estoit nécessaire par nécessité forcée que les princes du sang, comme estans astrains à un plus particulier devoir, et après eux la Noblesse et le peuple, missent la main vertueusement à l'œuvre, s'opposans par tous moyens, comme fidèles subjets et serviteurs, à telle ruine dudict royaume. ${ }^{23}$

9 La notion de nécessité est ici importante ; la trahison de ces chefs catholiques justifiant selon elle l'armement des protestants. Les arguments de Jeanne prennent, au fil du texte, une connotation de plus en plus grave. Toujours dans l'espoir de changer le cours des événements, elle ne manqua pas d'interpeller ses lecteurs sur le mépris auquel elle était confrontée conjointement à Charles IX, à son fils Henri de Navarre et au prince de Condé :

Et quant au service de Sa Majesté, estant son auctorité tellement méprisée et desdaignée qu'entre ces mutins infracteurs de ses Edicts et rebelles à ses commandemens, il ne tenoit plus rang de Roy; les princes de son sang chassez par les estrangers usurpateurs du lieu qu'ils debvoyent posséder. ${ }^{24}$

10 Cette félonie était explicitement assimilée à un crime de lèse-majesté quelques lignes plus loin, lorsque Jeanne expliqua que la couronne de Charles IX était sur le point de tomber au profit du clan adverse. La dénonciation de perfidie atteignit alors son paroxysme, justifiant d'autant plus la préparation au combat : qui pouvait laisser la royauté être ainsi usurpée? En habile conteuse, la mémorialiste préféra ne pas qualifier cet acte. L'accusation était plus lourde de sens si elle émanait du pouvoir en place, il valait bien mieux la suggérer. Aussi, Jeanne usa de questions oratoires et d'une métaphore préalable pour laisser le lecteur réfléchir et, évidemment, adhérer à son propos :

Car s'il est en nos moyens de tirer un homme de l'eau et nous le laissons noyer, ne sommes-nous pas meurtriers? Par plus forte raison, si nous voyons l'évident péril de ce royaume par la persécution de la religion, la couronne de nostre Roy estre à demy sur la teste de son ennemy, ne luy donnerions-nous pas une secousse pour achever de la renverser quand nous tiendrions les moyens que Dieu nous a donnez inutiles sans les déployer à venger tels actes ?25

11 Tout était dit alors, scellé. La seconde partie des Mémoires de Jeanne exhorte moins le clan catholique à ne pas attaquer qu'ils ne justifient une éventuelle attaque des protestants, ou tout au moins leur défense. Tout se présente comme si l'unique moyen d'éviter un affrontement résidait dans une disgrâce des Guise que seuls pouvaient provoquer Charles IX et Catherine de Médicis, principaux destinataires de l'œuvre. Jeanne annonça que sa décision était alors prise d'impliquer son héritier, Henri de Navarre et elle-même, dans le conflit. De surcroît, elle n'entendait pas être considérée par ceux qui la liraient comme une simple autorité morale du parti réformé, elle martela son souhait de s'investir pleinement. Elle qui n'avait pas participé aux premiers troubles et dont le fils ne s'était pas encore illustré au combat, signala sa détermination à travers la mise à disposition de tous les moyens dont elle disposait pour le bien de la cause protestante :

Je résolus donc de m'acheminer vers mon beau-frère avec mes enfans, et me joindre, de vie, biens et moyens, avec eux comme je l'avoy esté toujours de volonté, ne voulant tomber en la peine où nous avions esté aux pénultièmes troubles, ayans esté amusez et rendus inutiles par la malice d'aucuns de nos subjects, comme j'ay dict, et mesme mon fils, qu'il me faisoit grand mal de voir en cest aage parmy les femmes. ${ }^{26}$

12 La guerre parait inévitable ou, tout au moins, tel était le message que Jeanne souhaitait transmettre. Pourtant, l'argumentaire proposé dans ces Mémoires laisse entrevoir un 
élargissement des causes de celle-ci : les enjeux temporels du pouvoir semblent se mêler aux désaccords théologiques.

\section{Une mutation des intentions guerrières?}

13 Certes, la seconde partie des Mémoires est au cœur de l'argumentation choisie par Jeanne d'Albret. Plus dense, elle devait explicitement justifier l'armement des protestants qui se retranchaient à La Rochelle. Elle correspondait au message que la reine de Navarre souhaitait transmettre au pouvoir et au plus grand nombre. Pour autant, le reste du récit ne doit pas être négligé par notre analyse : en plus de resituer Jeanne dans son œuvre et dans son temps, ils nous renseignent plus largement sur les guerres civiles qui touchèrent le royaume.

a religion avait bien entendu une importance cruciale pour notre mémorialiste; immédiatement après les évocations sur la constance de sa foi, elle aborda celle de son fils Henri qui, selon elle, ne pouvait être le résultat de sa seule volonté :

Ce n'est pas par sa prudence, force ou constance : car l'aage de huict ans, qu'il avoit lors seulement accomplis, ne luy pouvoit apporter tout cela ; à Dieu seul donc en soit la gloire. ${ }^{27}$

Elle ignorait probablement la vérité : resté sous l'autorité de son père en mars 1562, Henri se laissa finalement conduire à la messe dès le mois de juin; il changea de religion de nombreuses fois au cours de sa vie jusqu'à sa dernière conversion en $1593^{28}$. Mais l'essentiel n'est pas là : la notion de prédestination, chère à Calvin et aux calvinistes et l'ancrage de la foi réformée dans l'esprit de notre mémorialiste sont ici évidents ${ }^{29}$. Les premières pages de l'œuvre font allusion à cette ferveur religieuse et, nous l'avons souligné, sa conversion constitue le point de départ de l'écriture du récit de sa vie ${ }^{30}$. La construction du récit laisse donc supposer que son engagement dans la troisième guerre, abordé en conclusion, fut motivé par sa récente conversion et induit des motivations religieuses.

Toutefois, il apparaît évident que la justification qu'incarnent ces Mémoires est avant tout politique. De par le contexte de la rédaction, l'effet escompté sur les destinataires et le thème, tout laisse supposer que la religion n'était pas le point de désaccord principal. En plus des accusations contre les Guise, la reine de Navarre brandit à de nombreuses reprises un étendard politique, faisant état du sort de ses sujets et soulignant en cela la haute opinion qu'elle avait de ses fonctions et le devoir qu'elle se faisait de les protéger :

Je m'en retournay avec mon fils chez moy en Béarn pour regarder à conduire les subjects que Dieu m'a donnez, et, aidée de sa grâce, empescher de tout mon pouvoir que c'est orage que je voyoy environner mon dict pays n'y entrast, en intention tous jours de servir à mon Dieu et à mon Roy. ${ }^{31}$

L'œuvre, dans son ensemble, apparaît ainsi comme étant particulièrement révélatrice des changements survenus après le second conflit ${ }^{32}$. Il s'agit du moment où les clans catholique et protestant s'organisèrent non plus seulement en groupes religieux mais bien en partis, avec pour chacun des ambitions politiques et des revendications sur le pouvoir. Le religieux n'était plus alors que le ciment qui liait les belligérants entre eux. Jeanne laissa percevoir ce changement en signifiant sa prochaine implication. Peu à peu, l'image de chef de clan, d'autorité morale et politique de Jeanne se dessine au fil de l'œuvre. Elle n'était pas n'importe quelle grande dame, elle était l'une des rares actrices possibles de la paix dans le royaume de France et pouvait engager l'ensemble de ses 
coreligionnaires dans les combats. Ce n'était pas la théologienne qui s'exprimait, mais la souveraine. Ce fut d'ailleurs en seigneur qu'elle évoqua la fidélité qui lui était dévolue sur ses terres :

Quant à mes vassaux, la plus grand part de ceux de la Guyenne, où nous avons presque tous nos biens, sont-ce pas eux qui nous y ont conduits? Les subjects, qui sont les Navarrois et les Béarnez [...], les uns sont, et en plus grand nombre que l'on ne pense et des deux religions, portant les armes avec mon fils, les autres sont demeurez au pays fermes à nos services, s'opposans fidèlement aux entreprinses que mes ennemis y veulent faire. ${ }^{33}$

Elle souligna également que son autorité était reconnue de tous : ne rappela-t-elle pas, à l'occasion, que Catherine de Médicis l'avait priée d'intervenir auprès de Condé pour le faire renoncer à ses entreprises guerrières $?^{34}$ Le récit que notre mémorialiste fit d'ellemême, se présentant comme la seule médiatrice possible entre Charles IX, Catherine de Médicis et les protestants, est particulièrement éloquent :

Car, pour le regard de leurs Majestés, j'avoy c'est honneur de leur appartenir de si près, joinct qu'ils avoyent tant cogneu en moy d'affection à leurs services et repos de ce royaume, que ce qui leur seroit proposé de ma part pour ceux de la religion, leur seroit hors de tout soubçon. De l'autre part que j'estoy en si bonne opinion entre ceux de ma religion, qu'ils se fieroyent bien en moy; et que par ce moyen estant près de leurs Majestés, et ayant la commodité d'aller visiter Monsieur le Prince [...] et Monsieur l'admiral, il me seroit facile, desmelant leurs méfiances d'un costé et d'autre, de rejoindre et approcher ces seigneurs de valeur de la personne du Roy. ${ }^{35}$

19 Nous retrouvons ici la détermination de la reine qui avait poussé Théodore Agrippa d'Aubigné à dire qu'elle était « une princesse, n'ayant de femme que le sexe, l'âme entière ès choses viriles, l'esprit puissant aux grandes affaires, le cœur invincible ès adversités $»^{36}$ . La reine n'hésita d'ailleurs pas à interpeller ses opposants dans une lamentation presque menaçante :

O pauvres misérables qui embrassez toutes ces grandeurs et promesses, et vous enflez d'une vaine espérance, serrez bien estroit ce contentement que recevez, cependant que la somme d'ignorance vous tient endormis; car, lorsque la vérité vous recueillera, tout ce bien vous eschappera et s'évanouira comme le songe. Il ne sera plus lors temps de vous repentir ; vostre deuil vous demeurera plus long que le bien qu'attendiez; regret marchera devant vous; honte tallonnera vos pas. Par le juste jugement de Dieu vostre conscience vous sera perpétuel bourreau. D'une et d'autre religion serez-vous mesprisez. ${ }^{37}$

Rédiger ses Mémoires est une manière de se raconter, de revenir sur son passé, de se placer dans la lumière. Il s'agissait également très souvent, pour les hommes et les femmes du XVI ${ }^{e}$ siècle, d'agir. Honorer une lignée, espérer un retour en grâce ou un changement de condition sont autant de motivations que nous retrouvons chez les mémorialistes du temps. Jeanne d'Albret ne fait pas ici figure d'exception. En tant que souveraine, elle pouvait s'attendre à ce que l'Histoire ne l'oubliât pas : il fallait avant tout justifier ses actes et la probable reprise des hostilités. Le récit s'apparente alors à une tribune, où la reine de Navarre ne pouvait légitimer son implication dans les conflits par des motifs ouvertement politiques. Ceci aurait consisté à agir exactement de la manière dont elle accusait la maison de Guise et à trahir le roi, ce dont elle s'était défendue plusieurs fois dans ses Mémoires. La religion et l'application de l'édit de Longjumeau devaient rester les seules motivations officielles des troubles. Ainsi, elle rédigea de manière fort habile un texte dans lequel la forme et le fond devaient convaincre. Le schéma argumentatif proposé permettait de rendre vraisemblables les arguments 
avancés : en faisant débuter son récit non pas par un engagement de vérité mais par son engagement religieux, Jeanne assurait le lecteur que l'armement protestant était toujours motivé par la foi. Il n'en demeure pas moins que les symptômes de la «maladie de la France $\rrbracket^{38}$ avaient changé, ce dont la reine de Navarre semble avoir eu pleinement conscience. C'est ainsi qu'elle prit finalement la tête du parti protestant en 1568, administra La Rochelle, communiqua avec les princes étrangers et s'engagea dans une guerre qui dura deux années.

\section{NOTES}

1. Les Ordonnances ecclésiastiques de Jeanne d'Albret avaient pour but de faire fuir le vice en Béarn, sous toutes ses formes. Il s'est notamment agi de traiter des abus d'alcool, des prostituées, ou de la danse. Voir J. Garrisson, Les protestants au XVI siècle, Paris, Fayard, 1988, p. 63-65. Voir également le chapitre «Ordonnances ecclésiastiques et confessionnalisation : le Béarn de Jeanne d'Albret et d'Henri de Navarre", dans V. Castagnet, O. Christin et N. Ghermani (dir.), Les affrontements religieux en Europe, du début $d u X^{X} I^{e}$ au milieu du XVII ${ }^{e}$ siècle, Villeneuve d'Ascq, Septentrion, 2008, p. 231-243.

2. Les Mémoires des Guerres de religion ont principalement été rédigés par des scripteurs masculins. Il s'agissait le plus souvent de justifier une participation, directe ou indirecte, aux conflits qui ont ravagé la France du second $\mathrm{XVI}^{\mathrm{e}}$ siècle. Or la guerre était, de fait, une affaire d'hommes. Nous n'avons recensé que trois femmes auteures de Mémoires au XVI ${ }^{\mathrm{e}}$ siècle ; il s'agit de Jeanne d'Albret, de Marguerite de Valois et de Charlotte Arbaleste.

3. Le manuscrit original des Mémoires a aujourd'hui disparu. Nous avons utilisé l'édition du baron de Ruble, publiée en 1893 chez Huart et Guillemin, qui s'avère être la première complète et commentée. Celle-ci présente, outre l'Ample déclaration, des poésies de la reine ainsi que des pièces justificatives, notamment une partie de sa correspondance. Enfin, soulignons que l'éditeur a inséré tout au long de l'œuvre de nombreuses notes qui éclairent le lecteur ainsi qu'un sommaire détaillé, organisé par année. J. d'Albret, Mémoires et Poésies, éd. Ruble, Paris, Huart, 1893, 240 pages, in-8.

4. Eliane Viennot considère par exemple que le récit est un mémoire justificatif, autrement dit un texte destiné à être divulgué immédiatement dans le but de faire infléchir le cours de la vie politique. Sans remettre en cause cette affirmation, nous considérons que bien d'autres Mémoires du XVI e avaient la même vocation. Il s'agissait souvent d'affirmer ne pas souhaiter être lu comme gage de sincérité, tout en faisant connaître à un cercle restreint ses écrits, en espérant un changement de condition. Voir Marguerite de Valois, Mémoires et Discours, éd. Eliane Viennot, Saint-Etienne, PUSE, 2004, p. 27.

5. Cet élément est évoqué par N. Kuperty-Tsur dans son article « Jeanne d'Albret ou la persuasion par la passion », dans E. Berriot-Salvadore, P. Chareyre et C. Martin-Ulrich (dir.), Jeanne d'Albret et sa cour, Actes du colloque international de Pau (17-19 mai 2001), Paris, Honoré Champion, 2004, p. 259-280. Pour une vision d'ensemble du genre des Mémoires au XVI ${ }^{\mathrm{e}}$ siècle, voir N. KupertyTsur, Se dire à la Renaissance. Les Mémoires au XVI siècle, Paris, J. Vrin, 1997.

6. Après avoir pris la tête du mouvement protestant en 1568, Jeanne se réfugia à La Rochelle et tenta de légitimer sa prise d'armes. Elle écrivit notamment à Charles IX, à Catherine de Médicis, au duc d'Anjou, au cardinal de Bourbon et à Élisabeth d'Angleterre. Claudie Martin-Ulrich 
explique que les lettres et les Mémoires de Jeanne d'Albret entretiennent « un rapport de complémentarité, dans lequel ces derniers donneront l'occasion d'une amplification et d'un développement impossibles dans l'espace d'une lettre ", voir son article «Catherine de Médicis et Jeanne d'Albret, la reine mère et la reine conteuse ", dans I. Cogitore et F. Goyet (dir.), Devenir roi. Essais sur la littérature adressée au prince, Grenoble, ELLUG, 2001, p. 224. L'ensemble de l'œuvre de Jeanne d'Albret a également été récemment étudié par Nadine Kuperty-Tsur, apportant un éclairage sur le message véhiculé par ses Mémoires, dans l'article «Hardie et ardente : pérennité de l'ethos de Jeanne d'Albret au miroir de son œuvre ", dans M. Tsimbidy et F. Charbonneau (dir.), Les mémorialistes en leurs écrits, Paris, Garnier, 2014.

7. J. d'Albret, Mémoires..., cit., p. 117. Outre les quelques mentions à l'adverbe « icy » pour parler de son texte, Jeanne d'Albret utilise une fois le mot "traité ", qui évoque un récit au thème particulier.

8. Ibid., p. 1.

9. Ibid., p. 2.

10. Ibid., p. 5.

11. À ce propos, voir l'article d'E. Lesne, «Les Mémoires et leurs destinataires dans la seconde moitié du XVII ${ }^{e}$ siècle", dans M. Bertaud et F.-X. Cuche (dir.), Le genre des Mémoires, essai de définition, Paris, Klincksieck, 1995, p. 27-44.

12. Le découpage que nous proposons ici concerne les questions religieuses de la page 1 à la page 6 ; le retour sur les perfidies de la maison de Guise et la justification des combats de la page 6 à la page 107 ; la décision de partir pour La Rochelle ainsi que ce voyage de la page 80 à la page 120 ; le retour au présent et la théorisation de son œuvre des pages 120 à 121 .

13. À propos de la conversion de la reine de Navarre, voir l'ouvrage de D. Bryson, Queen Jane and the promised Land. Dynasty, Homeland and Violence in Sixteenth century France, Cologne, Brill, 1999, p. 77-114.

14. J. d'Albret, Mémoires..., cit., p. 2-3.

15. Antoine de Bourbon changea de nombreuses fois de religion, au gré des nécessités politiques. En ces temps de troubles du royaume de France, l'instabilité religieuse était très fréquente ; le cas de Jeanne fait ici figure d'exception. Voir l'ouvrage de R. Lambin, Femmes de paix. La coexistence religieuse et les dames de la noblesse en France 1520-1630, Paris, L'Harmattan, 2003, p. 15.

16. L'œuvre couvre huit années de la vie de la reine de Navarre, de 1560 à 1568.

17. J. d'Albret, Mémoires..., cit., p. 121.

18. Le pacte autobiographique est l'engagement que prend l'auteur de raconter directement sa vie, dans un esprit de vérité. À propos de cette notion, voir P. Lejeune, Le pacte autobiographique [1975], Paris, Seuil, 1996.

19. N. Kuperty-Tsur, «La stratégie des préfaces dans les Mémoires du XVI ${ }^{\mathrm{e}}$ siècle », dans M. Bertaud et F.-X. Cuche (dir.), Le genre des Mémoires..., cit., p. 13.

20. À propos des enjeux politiques de la période, voir l'ouvrage de référence d'A. Jouanna, Le devoir de révolte. La noblesse française et la gestation de l'État moderne, 1559-1661, Paris, Fayard, 1989, notamment les pages 150-152.

21. J. d'Albret, Mémoires..., cit., p. 6-7. La surprise de Meaux était une conspiration menée par le prince de Condé pour enlever le roi Charles IX et la reine Catherine de Médicis. Il s'agissait de les soustraire à l'influence de la maison de Guise. Voir D. El Kenz et C. Gantet, Guerres et paix de religion en Europe, $\mathrm{XVI}^{e}-\mathrm{XVII}{ }^{e}$ siècles, Paris, Armand Colin, 2003, p. 84.

22. Ibid., p. 47.

23. Ibid., p. 73.

24. Ibid., p. 79.

25. Ibid., p. 80.

26. Ibid., p. 80-81.

27. J. d'Albret, Mémoires..., cit., p. 4. 
28. Henri abjura finalement sa foi protestante le 25 juillet 1593 afin de réconcilier les Français, mettre fin aux Guerres de religion et assurer sa légitimité à ceindre la couronne. Il s'agissait alors de sa sixième conversion.

29. À propos de la notion de prédestination au XVI siècle, voir J. Garrisson, Les protestants au XVI siècle..., cit., p. 30.

30. Les liens particuliers que les femmes $d u X I^{e}$ siècle entretinrent, notamment dans les rangs de la noblesse, sont bien connus depuis les travaux de Nancy Roeckler et Natalie Zemon Davis. Voir notamment N. Roeckler, "Les femmes de la noblesse huguenote au XVI ${ }^{\mathrm{e}}$ siècle ", dans L'Amiral de Coligny et son temps, Actes du colloque de Paris, 24-28 octobre 1972, Paris, Société de l'histoire du protestantisme français, 1974, p. 227-250 ; N. Zimon Davis, Society and culture in early modern France, Stanford, SUP, 1975.

31. J. d'Albret, Mémoires..., cit., p. 86-87. Rappelons ici, si besoin est, que Jeanne d'Albret était souveraine d'un royaume qui ignorait la loi salique. Celle-ci, censée avoir été rédigée sous le règne de Clovis, évinçait les femmes de la succession royale de France. Voir E. Viennot, La France, les femmes et le pouvoir. L'invention de la loi salique, Paris, Perrin, 2006.

32. Un grand nombre de libelles justificatifs avaient accompagné la prise d'armes de 1567, critiquant le gouvernement du royaume. L'absence de fidélité aux engagements pris était très souvent mise en avant. Voir A. Jouanna, J. Boucher, D. Biloghi, G. Le Thiec (dir.), Histoire et dictionnaire des Guerres de religion, Paris, Robert Laffont, 1998, p. 165-169.

33. Ibid., p. 96-97.

34. Ibid., p. 23.

35. Ibid., p. 56-57.

36. Th. Agrippa d'Aubigné, Histoire Universelle, Paris Renouard, 1886, tome III, p. 291.

37. J. d'Albret, Mémoires..., cit., p. 83.

38. Ibid., p. 75.

\section{RÉSUMÉS}

Les Mémoires de Jeanne d'Albret ont été rédigés après l'irritation provoquée par la paix de Longjumeau en 1568. La reine de Navarre rédigea alors, à l'attention de Catherine de Médicis et du roi Charles IX, le récit d'une partie de sa vie, proposé comme un violent réquisitoire à l'encontre de ses ennemis.

En tant que fervente protestante et autorité morale de ses coreligionnaires, Jeanne agit par la plume. Il s'agissait de justifier son implication nouvelle dans les conflits et de légitimer l'armement des protestants. Ce texte, aux choix rédactionnels singuliers, porte en lui le changement survenu après le second conflit dans le royaume de France : la politisation des affrontements.

INDEX

Mots-clés : Mémoires, d'Albret (Jeanne), guerre de religion, apologétisme, écriture protestante 